\title{
Solution of $(3+1)$-Dimensional Nonlinear Cubic Schrodinger Equation by Differential Transform Method
}

\author{
Hassan A. Zedan ${ }^{1,2}$ and M. Ali Alghamdi ${ }^{1}$ \\ ${ }^{1}$ Mathematics Department, Faculty of Science, King Abdulaziz University, P.O. Box 80203, Jeddah 21589, \\ Saudi Arabia \\ ${ }^{2}$ Department of Mathematics, Faculty of Science, Kafr El-Sheikh University, Kafr El-Sheikh 33516, Egypt
}

Correspondence should be addressed to Hassan A. Zedan, hassanzedan2003@yahoo.com

Received 15 September 2011; Accepted 8 December 2011

Academic Editor: Xing-Gang Yan

Copyright (c) 2012 H. A. Zedan and M. A. Alghamdi. This is an open access article distributed under the Creative Commons Attribution License, which permits unrestricted use, distribution, and reproduction in any medium, provided the original work is properly cited.

Four-dimensional differential transform method has been introduced and fundamental theorems have been defined for the first time. Moreover, as an application of four-dimensional differential transform, exact solutions of nonlinear system of partial differential equations have been investigated. The results of the present method are compared very well with analytical solution of the system. Differential transform method can easily be applied to linear or nonlinear problems and reduces the size of computational work. With this method, exact solutions may be obtained without any need of cumbersome work, and it is a useful tool for analytical and numerical solutions.

\section{Introduction}

In this paper, we study system of nonlinear partial differential equations (PDEs). In order to solve systems of differential equations, the commonly used methods are the method of characteristics and the Riemann invariants among other methods. The existing techniques have difficulties in related to the size of computational work, especially when the system has several PDEs. More recently, Adomian decomposition method, were used to handle systems of PDEs by [1], and here we present an alternative method for the system. The concept of differential transform was first introduced by Zhou [2], who solved linear and nonlinear initial value problems in electric circuit analysis. The transformation method, called threedimensional differential transform, is different from the high-order Taylor series method, which consists of computing the coefficients of the Taylor series of the solution using the initial data and the PDE. But the Taylor series method requires more computational work for large orders. The present method is well addressed in [3-7]. The differential transform 
technique is an iterative procedure for obtaining Taylor series solutions of differential equations and systems of PDEs. This method reduces the size of computational domain and is applicable to many problems easily. In the present work, in order to extend applications of the differential transform method to different problems, four-dimensional differential transform have been defined and new theorems have been prooved. In this paper, Differential Transformation Method (DTM) is employed to obtain the solution of the (3+1)-dimensional nonlinear cubic Schrödinger equation

$$
i \psi_{t}+\psi_{x x}+\psi_{y y}+\psi_{z z}+\psi\left(|\psi|^{2}-S(x, y, z, t)\right)=0 .
$$

We put

$$
\psi=u+i v
$$

From (1.1) we obtain that

$$
\begin{gathered}
M(u, v)=-v_{t}+u_{x x}+u_{y y}+u_{z z}+u\left(u^{2}+v^{2}-S(x, y, z, t)\right)=0 \\
N(u, v)=u_{t}+v_{x x}+v_{y y}+v_{z z}+v\left(u^{2}+v^{2}-S(x, y, z, t)\right)=0 .
\end{gathered}
$$

\section{Basic Idea of the Differential Equation}

\subsection{One-Dimensional Differential Transform Method}

We introduce in this section the basic definition of the one-dimensional differential transformation.

Definition 2.1. If $u(t)$ is analytic in the domain $T$, then it will be differentiated continuously with respect to time $t$ :

$$
\frac{d^{k} u(t)}{d t^{k}}=\phi(t, k), \quad \forall t \in T
$$

for $t=t_{i}$, where $\phi(t, k)=\phi\left(t_{i}, k\right)$, and $k$ and $k$ belong to the set of nonnegative integer denoted as the $K$ domain.

Therefore, (1.2) can be written as

$$
U_{i}(k)=\phi\left(t_{i}, k\right)=\left[\frac{d^{k} u(t)}{d t^{k}}\right]_{t=t_{i}}, \quad \forall k \in K
$$

where $U_{i}(k)$ is called spectrum of $u(t)$ at $t=t_{i}$, in the $K$ domain.

Definition 2.2. If $u(t)$ can be expressed by Taylor's series, then $u(t)$ can be represented as

$$
u(t)=\sum_{k=0}^{\infty} \frac{\left(t=t_{i}\right)}{k !} U(k)
$$


Equation (2.3) is known as the inverts transformation of $U(k)$. If $U(k)$ is defined as

$$
U(k)=M(k)\left[\frac{d^{k} q(t) u(t)}{d t^{k}}\right]_{t=t_{i}},
$$

where $k=0,1,2, \ldots, \infty$, then the function $u(t)$ can be described as

$$
u(t)=\frac{1}{q(t)} \sum_{k=0}^{\infty} \frac{\left(t-t_{i}\right)}{k !} \frac{U(k)}{M(k)}
$$

where $M(k) \neq 0, q(t) \neq 0$. The function $M(k)$ is called the weighting factor and $q(t)$ is regarded as kernel corresponding to $u(t)$. If $M(k)=1$ and $q(t)=1$, then (1.3) and (2.2) are equivalent. In this way, (2.1) can be treated as a special case of (2.3). In this paper, the transformation with $M(k)=1 / k !$ and $q(t)=1$ is applied.

Then (2.4) becomes

$$
U(k)=\left[\frac{d^{k} u(t)}{d t^{k}}\right]_{t=t_{i}}, \quad \text { where } k=0,1,2, \ldots, \infty
$$

Using the differential transform, a differential equation in the domain of interest can be transformed to be an algebraic equation in the $K$ domain and $u(t)$ can be obtained by finiteterm Taylor series plus a remainder, as

$$
u(t)=\frac{1}{q(t)} \sum_{k=0}^{\infty} \frac{\left(t-t_{i}\right)}{k !} \frac{U(k)}{M(k)}+R_{n+1}(t)=\sum_{k=0}^{\infty}\left(t-t_{i}\right)^{k} U(k)+R_{n+1}(t) .
$$

In order to speed up the convergent rate and the accuracy of calculation, the entire domain of $t$ needs to be split into subdomains.

\subsection{Two-Dimensional Differential Transform Method}

Definition 2.3. Given an $w$ function which has two components such as $x, t$ to $w$-dimensional differential transform of $w(x, t)$ is defined

$$
W(k, h)=\frac{1}{k ! h !}\left[\frac{\partial^{k+h} W(x, t)}{\partial x^{k} \partial y^{h}}\right]_{(0,0)},
$$

where $W(x, y)$ the original is function and $W(k, h)$ is the transformed function. Again, the transformation can be called $T$-function, and the lower case and upper case letters represent the original and transformed functions, respectively (Table 1).

Definition 2.4. The differential inverse transform of $W(k, h)$ is defined as

$$
W(x, t)=\sum_{k=0}^{\infty} \sum_{h=0}^{\infty} W(k, h) x^{k} t^{h}
$$


Table 1: The fundamental theorems.

\begin{tabular}{ll}
\hline Original function & Transformed function \\
\hline (1) $W(x, t)=u(x, t) \pm v(x, t)$, & $W(k, h)=U(k, h) \pm V(k, h)$. \\
(2) $W(x, t)=\lambda u(x, t)$, & $W(k, h)=\lambda U(k, h), \lambda$ is constant \\
(3) $W(x, t)=\partial u(x, t) / \partial x$, & $W(k, h)=(k+1) U(k+1, h)$ \\
(4) $W(x, t)=\partial u(x, t) / \partial t$ & $W(k, h)=(h+1) U(k, h+1)$ \\
(5) $W(x, t)=\partial^{r+s} u(x, t) / \partial x^{r} \partial t^{s}$ & $W(k, h)=(k+1)(k+2) \cdots(k+r)(h+1)$ \\
& $\times(h+2) \cdots(h+s) U(k+r, h+s)$ \\
(6) $W(x, t)=u(x, t) v(x, t)$ & $W(k, h)=\sum_{r=0}^{k} \sum_{s=0}^{k} U(r, h-s) V(k-r, s)$ \\
(7) $W(x, t)=(\partial u(x, t) / \partial x)(\partial v(x, t) / \partial x)$, & $W(k, h)=\sum_{k=0}^{\infty} \sum_{h=0}^{\infty}(r+1)(k-r+1) U(r+1, h-s)$ \\
(8) $W(x, t)=u(x, t)(\partial u(x, t) / \partial x)$, & $W(k, h)=\sum_{k=0}^{\infty} \sum_{h=0}^{\infty}(k-r+1) U(r, h-s) U(k-r+1, s)$ \\
\hline
\end{tabular}

and from (2.8) and (2.9) can be concluded as follows:

$$
W(x, t)=\sum_{k=0}^{\infty} \sum_{h=0}^{\infty} \frac{1}{k ! h !}\left[\frac{\partial^{k+h} W(x, t)}{\partial x^{k} \partial t^{h}}\right]_{(0,0)} x^{k} t^{h} .
$$

\subsection{Three-Dimensional Differential Transform}

Definition 2.5. Given an $w$ function which has three components such as $x, y, t$. Threedimensional differential transform of $w(x, y, t)$ is defined as

$$
W(k, h, m)=\frac{1}{k ! h ! m !}\left[\frac{\partial^{h+k+m} w(x, y, t)}{\partial x^{k} \partial y^{h} \partial t^{m}}\right]_{(0,0,0)}
$$

where $w(x, y, t)$ is the original function and $W(k, h, m)$ is the transformed function. Again, the transformation can be called $T$-function, and the lower case and upper case letters represent the original and transformed functions, respectively (Table 2).

Definition 2.6. The differential inverse transform of $W(k, h, m)$ is defined as

$$
w(x, y, t)=\sum_{k=0}^{\infty} \sum_{h=0}^{\infty} \sum_{m=0}^{\infty} W(k, h, m) x^{k} y^{h} t^{m}
$$

and from (2.11) and (2.12) can be concluded as

$$
w(x, y, t)=\sum_{k=0}^{\infty} \sum_{h=0}^{\infty} \sum_{m=0}^{\infty} \frac{1}{k ! h ! m !}\left[\frac{\partial^{h+k+m} w(x, y, t)}{\partial x^{k} \partial y^{h} \partial t^{m}}\right]_{(0,0,0)} x^{k} y^{h} t^{m}
$$


Table 2: Fundamental theorems for three-dimensional case.

\begin{tabular}{|c|c|}
\hline Original function & Transformed function \\
\hline (1) $w(x, y, t)=u(x, y, t) \pm v(x, y, t)$ & $W(h, k, m)=U(k, h, m) \pm V(k, h, m)$ \\
\hline (2) $w(x, y, t)=c u(x, y, t)$ & $W(h, k, m)=c U(k, h, m)$ \\
\hline (3) $w(x, y, t)=\partial u(x, y, t) / \partial x$ & $W(h, k, m)=(k+1) U(k+1, h, m)$ \\
\hline (4) $w(x, y, t)=\partial u(x, y, t) / \partial y$ & $W(h, k, m)=(h+1) U(k, h+1, m)$ \\
\hline (5) $w(x, y, t)=\partial u(x, y, t) / \partial t$ & $W(h, k, m)=(m+1) U(k, h, m+1)$ \\
\hline (6) $w(x, y, t)=\partial^{r+s+p} u(x, y, t) / \partial x^{r} \partial y^{s} \partial t^{p}$ & $\begin{aligned} W(h, k, m)= & (k+1)(k+2) \cdots(k+r) \times(h+2) \cdots \\
& (h+s)(m+1)(m+2) \cdots(m+p)\end{aligned}$ \\
\hline (7) $w(x, y, t)=u(x, y, t) v(x, y, t)$ & $W(h, k, m)=\sum_{r=0}^{k} \sum_{s=0}^{h} \sum_{p=0}^{m} U(r, h-s, m-p) V(k-r, s, p)$ \\
\hline (8) $w(x, y, t)=(\partial u(x, y, t) / \partial x)(\partial v(x, y, t) / \partial y)$ & $\begin{aligned} W(h, k, m)= & \sum_{r=0}^{k} \sum_{s=0}^{h} \sum_{p=0}^{m}(k-r+1)(h-s+1) \\
& \times U(k-r+1, s, p) \times V(r, h-s+1, m-p)\end{aligned}$ \\
\hline
\end{tabular}

Table 3: Fundamental theorems for four-dimensional case.

\begin{tabular}{|c|c|}
\hline Original function & Transformed function \\
\hline (1) $w(x, y, z, t)=u(x, y, z, t) \pm v(x, y, z, t)$ & $W(h, k, m, n)=U(k, h, m, n) \pm V(k, h, m, n)$ \\
\hline (2) $w(x, y, z, t)=c u(x, y, z, t)$ & $W(h, k, m, n)=c U(k, h, m, n)$ \\
\hline $\begin{array}{c}\text { (3) } w(x, y, z, t)=\partial^{r+s+p+q} u(x, y, z, t) / \\
\partial x^{r} \partial y^{s} \partial z^{p} \partial t^{q}\end{array}$ & $\begin{array}{c}W(h, k, m, n)=(k+1)(k+2) \cdots(k+r)(h+1)(h+2) \cdots \\
(h+s)(m+1)(m+2) \times \cdots(m+p)(n+1)(n+2) \cdots \\
(n+q) U(k+r, h+s, m+p, n+q)\end{array}$ \\
\hline (4) $w(x, y, z, t)=\partial u(x, y, z, t) / \partial t$ & $W(h, k, m, n)=(m+1) U(k, h, m, n+1)$ \\
\hline (5) $w(x, y, z, t)=u(x, y, z, t) v(x, y, z, t)$ & $\begin{array}{l}W(h, k, m, n)=\sum_{r=0}^{k} \sum_{s=0}^{h} \sum_{p=0}^{m} \sum_{q=0}^{n} \\
U(r, h-s, m-p, n-q) V(k-r, s, p, q)\end{array}$ \\
\hline
\end{tabular}

\subsection{Four-Dimensional Differential Transform}

Definition 2.7. Given an $w$ function which has four components such as $x, y, z$, t. Fourdimensional differential transform of $w(x, y, z, t)$ is defined as

$$
W(k, h, m, n)=\frac{1}{k ! h ! m ! n !}\left[\frac{\partial^{h+k+m+n} w(x, y, z, t)}{\partial x^{k} \partial y^{h} \partial z^{m} \partial t^{n}}\right]_{(0,0,0,0)},
$$

where $w(x, y, z, t)$ is the original function and $W(k, h, m, n)$ is the transformed function. Again, the transformation can be called $T$-function, and the lower case and upper case letters represent the original and transformed functions, respectively (Table 3 ).

Definition 2.8. The differential inverse transform of $W(k, h, m, n)$ is defined as

$$
w(x, y, z, t)=\sum_{k=0}^{\infty} \sum_{h=0}^{\infty} \sum_{m=0}^{\infty} \sum_{n=0}^{\infty} W(k, h, m, n) x^{k} y^{h} z^{m} t^{n}
$$

and from (2.14) and (2.15) can be concluded as

$$
w(x, y, z, t)=\sum_{k=0}^{\infty} \sum_{h=0}^{\infty} \sum_{m=0}^{\infty} \sum_{n=0}^{\infty} \frac{1}{k ! h ! m ! n !}\left[\frac{\partial^{h+k+m} w(x, y, t)}{\partial x^{k} \partial y^{h} \partial t^{m}}\right]_{(0,0,0,0)} x^{k} y^{h} z^{m} t^{n}
$$


Theorem 2.9. If $w(x, y, z, t)=u(x, y, z, t) v(x, y, z, t) \omega(x, y, z, t)$, then

$$
\begin{aligned}
W(h, k, m, n)= & \sum_{r=0}^{k} \sum_{t=0}^{k-r} \sum_{s=0}^{h} \sum_{i=0}^{h-s} \sum_{p=0}^{m} \sum_{j=0}^{m-p} \sum_{q=0}^{n} \sum_{a=0}^{n-q} U[r, s, p, n-q-a] V[t, i, j, q] \\
& \times \mathbb{W}[k-r-t, h-s-i, m-p-j, a] .
\end{aligned}
$$

Proof. From the definition of transform,

$$
\begin{aligned}
W(0,0,0,0)= & U[0,0,0,0] V[0,0,0,0] \omega[0,0,0,0] \\
W(1,0,0,0)= & U[1,0,0,0] V[0,0,0,0] \omega[0,0,0,0]+U[0,0,0,0] V[1,0,0,0] \omega[0,0,0,0] \\
& +U[0,0,0,0] V[0,0,0,0] \omega[1,0,0,0], \\
W(2,0,0,0)= & U[2,0,0,0] V[0,0,0,0] \omega[0,0,0,0]+U[1,0,0,0] V[1,0,0,0] \omega[0,0,0,0] \\
& +U[0,0,0,0] V[2,0,0,0] \omega[0,0,0,0]+U[1,0,0,0] V[0,0,0,0] \omega[1,0,0,0] \\
& +U[0,0,0,0] V[1,0,0,0] \omega[1,0,0,0]+U[0,0,0,0] V[0,0,0,0] \omega[2,0,0,0], \\
W(0,1,0,0)= & U[0,1,0,0] V[0,0,0,0] \omega[0,0,0,0]+U[0,0,0,0] V[0,1,0,0] \omega[0,0,0,0] \\
& +U[0,0,0,0] V[0,0,0,0] \omega[0,1,0,0], \\
W(0,2,0,0)= & U[0,2,0,0] V[0,0,0,0] \omega[0,0,0,0]+U[0,1,0,0] V[0,1,0,0] \omega[0,0,0,0] \\
& +U[0,0,0,0] V[0,2,0,0] \omega[0,0,0,0]+\cdots, \\
W(0,0,1,0)= & U[0,0,1,0] V[0,0,0,0] \omega[0,0,0,0]+U[0,0,0,0] V[0,0,1,0] \omega[0,0,0,0] \\
& +U[0,0,0,0] V[0,0,0,0] \omega[0,0,1,0] .
\end{aligned}
$$

In general, we have

$$
\begin{aligned}
W(h, k, m, n)= & \sum_{r=0}^{k} \sum_{t=0}^{k-r} \sum_{s=0}^{h} \sum_{i=0}^{h-s} \sum_{p=0}^{m} \sum_{j=0}^{m-p} \sum_{q=0}^{n} \sum_{a=0}^{n-q} U[r, s, p, n-q-a] V[t, i, j, q] \\
& \times \mathbb{W}[k-r-t, h-s-i, m-p-j, a] .
\end{aligned}
$$

\section{Analysis of Method}

To investigate the solution of (1.3), we first construct a system by differential transformation method as follows:

$$
\begin{aligned}
(n+1) V[k, h, m, n+1]= & (k+1)(k+2) U[k+2, h, m, n]+(h+1)(h+2) U[k, h+2, m, n] \\
& +(m+1)(m+2) U[k, h, m+2, n] \\
& +\sum_{r=0}^{k} \sum_{t=0}^{k-r} \sum_{s=0}^{h} \sum_{i=0}^{h-s} \sum_{p=0}^{m} \sum_{j=0}^{m-p} \sum_{q=0}^{n} \sum_{a=0}^{n-a} U[r, s, p, n-q-a] U[t, i, j, q] \\
& \times U[k-r-t, h-s-i, m-p-j, a]
\end{aligned}
$$


Mathematical Problems in Engineering

$$
\begin{aligned}
& +\sum_{r=0}^{k} \sum_{t=0}^{k-r} \sum_{s=0}^{h} \sum_{i=0}^{h-s} \sum_{p=0}^{m} \sum_{j=0}^{m-p} \sum_{q=0}^{n} \sum_{a=0}^{n-a} U[r, s, p, n-q-a] V[t, i, j, q] \\
& \times V[k-r-t, h-s-i, m-p-j, a] \\
& -\sum_{r=0}^{k} \sum_{s=0}^{h} \sum_{p=0}^{m} \sum_{q=0}^{p} U[r, h-s, m-p, n-q] S[k-r, s, p, q], \\
(n+1) U[k, h, m, n+1]= & -(k+1)(k+2) V[k+2, h, m, n]-(h+1)(h+2) V[k, h+2, m, n] \\
& -(m+1)(m+2) V[k, h, m+2, n] \\
& -\sum_{r=0}^{k} \sum_{t=0}^{k-r} \sum_{s=0}^{h} \sum_{i=0}^{h-s} \sum_{p=0}^{m} \sum_{j=0}^{m-p} \sum_{q=0}^{n} \sum_{a=0}^{n-a} U[r, s, p, n-q-a] U[t, i, j, q] \\
& \times V[k-r-t, h-s-i, m-p-j, a] \\
& -\sum_{r=0}^{k} \sum_{t=0}^{k-r} \sum_{s=0}^{h} \sum_{i=0}^{h-s} \sum_{p=0}^{m} \sum_{j=0}^{m-p} \sum_{q=0}^{n} \sum_{a=0}^{n-a} V[r, s, p, n-q-a] V[t, i, j, q] \\
& \times V[k-r-t, h-s-i, m-p-j, a] \\
& +\sum_{r=0}^{k} \sum_{s=0}^{h} \sum_{p=0}^{m} \sum_{q=0}^{p} V[r, h-s, m-p, n-q] S[k-r, s, p, q] .
\end{aligned}
$$

Subject to Taylor's expand of initial condition

$$
\begin{aligned}
u(x, y, z, 0) & =G(x, y, z)=\sum_{k=0}^{\infty} \sum_{h=0}^{\infty} \sum_{m=0}^{\infty} U(k, h, m) x^{k} y^{h} z^{m} \\
& =F(0,0,0)+\sum_{k=0}^{\infty} \sum_{h=0}^{\infty} \sum_{m=0}^{\infty} \frac{F^{(k+h+m)}(0,0,0)}{k ! h ! m !} x^{k} y^{h} z^{m}, \\
v(x, y, z, 0) & =G(x, y, z)=\sum_{k=0}^{\infty} \sum_{h=0}^{\infty} \sum_{m=0}^{\infty} V(k, h, m) x^{k} y^{h} z^{m} \\
& =G(0,0,0)+\sum_{k=0}^{\infty} \sum_{h=0}^{\infty} \sum_{m=0}^{\infty} \frac{G^{(k+h+m)}(0,0,0)}{k ! h ! m !} x^{k} y^{h} z^{m} .
\end{aligned}
$$

Substituting (3.2) into (3.1) and using operation of Table 3, we get

$$
\begin{aligned}
& V[k, h, m, n+1] \\
& \begin{aligned}
=\frac{1}{(n+1)}\left[\begin{array}{r}
(k \\
+
\end{array}\right. & 1)(k+2) U[k+2, h, m, n]+(h+1)(h+2) U[k, h+2, m, n] \\
& +(m+1)(m+2) U[k, h, m+2, n] \\
& +\sum_{r=0}^{k} \sum_{t=0}^{k-r} \sum_{s=0}^{h} \sum_{i=0}^{h-s} \sum_{p=0}^{m} \sum_{j=0}^{m-p} \sum_{q=0}^{n} \sum_{a=0}^{n-a} U[r, s, p, n-q-a] U[t, i, j, q]
\end{aligned}
\end{aligned}
$$




$$
\begin{aligned}
& \times U[k-r-t, h-s-i, m-p-j, a] \\
& +\sum_{r=0}^{k} \sum_{t=0}^{k-r} \sum_{s=0}^{h} \sum_{i=0}^{h-s} \sum_{p=0}^{m} \sum_{j=0}^{m-p} \sum_{q=0}^{n} \sum_{a=0}^{n-a} U[r, s, p, n-q-a] V[t, i, j, q] \\
& \times V[k-r-t, h-s-i, m-p-j, a] \\
& \left.-\sum_{r=0}^{k} \sum_{s=0}^{h} \sum_{p=0}^{m} \sum_{q=0}^{p} U[r, h-s, m-p, n-q] S[k-r, s, p, q]\right]
\end{aligned}
$$

$U[k, h, m, n+1]$

$$
\begin{aligned}
\frac{1}{(n+1)}[- & (k+1)(k+2) V[k+2, h, m, n]-(h+1)(h+2) V[k, h+2, m, n] \\
& -(m+1)(m+2) V[k, h, m+2, n] \\
& -\sum_{r=0}^{k} \sum_{t=0}^{k-r} \sum_{s=0}^{h} \sum_{i=0}^{h-s} \sum_{p=0}^{m} \sum_{j=0}^{m-p} \sum_{q=0}^{n} \sum_{a=0}^{n-a} U[r, s, p, n-q-a] U[t, i, j, q] \\
& \times V[k-r-t, h-s-i, m-p-j, a] \\
& -\sum_{r=0}^{k} \sum_{t=0}^{k-r} \sum_{s=0}^{h} \sum_{i=0}^{h-s} \sum_{p=0}^{m} \sum_{j=0}^{m-p} \sum_{q=0}^{n} \sum_{a=0}^{n-a} V[r, s, p, n-q-a] V[t, i, j, q] \\
& \times V[k-r-t, h-s-i, m-p-j, a] \\
& \left.+\sum_{r=0}^{k} \sum_{s=0}^{h} \sum_{p=0}^{m} \sum_{q=0}^{p} V[r, h-s, m-p, n-q] S[k-r, s, p, q]\right] .
\end{aligned}
$$

In order to obtain the unknowns of $U(k, h, m, n)$ and $V(k, h, m, n), k, h, m, n=0,1,2, \ldots$, we must construct and solve the above equation and substitute in (2.16).

\section{Application}

In this section, the differential transformation technique is applied to solve (1.3). This method only needs the initial condition of covering PDEs. Firstly, we consider the solution of (1.3) with the initial conditions:

$$
\begin{gathered}
u(x, y, z, 0)=1+\operatorname{Tanh}(x+y+z), \\
v(x, y, z, 0)=1-\operatorname{Tanh}(x+y+z), \\
S(x, y, z, 0)=-4+8 \operatorname{Tanh}(x+y+z)^{2} .
\end{gathered}
$$


Suppose that $x_{0}=y_{0}=z_{0}=t_{0}=0$, in Definition 2.7, then we have

$$
\begin{array}{ccc}
U[0,0,0,0]=1, & U[1,0,0,0]=1, & U[2,0,0,0]=0, \\
U[3,0,0,0]=-\frac{1}{3}, \quad U[4,0,0,0]=0, & U[5,0,0,0]=\frac{2}{15}, \\
U[0,1,0,0]=1, & U[0,0,2,0]=0, & U[0,0,3,0]=-\frac{1}{3}, \\
U[2,3,0,0]=\frac{4}{3}, \quad U[0,0,4,0]=0, \quad U[0,0,5,0]=\frac{2}{15}, \quad U[2,1,0,0]=-1, \\
U[4,1,0,0]=\frac{2}{3}, \quad U[1,2,0,0]=-1, \quad U[0,2,0,0]=0, \\
U[0,3,0,0]=-\frac{1}{3}, \quad U[0,4,0,0]=0, \quad U[0,5,0,0]=\frac{2}{15}, \\
U[0,0,1,0]=1, \quad U[4,3,0,0]=-\frac{17}{9}, \quad U[1,4,0,0]=\frac{2}{3}, \\
U[3,4,0,0]=-\frac{17}{9}, \quad U[2,0,1,0]=-1, \quad U[4,0,1,0]=\frac{2}{3}, \\
U[3,0,2,0]=\frac{4}{3}, \quad U[4,0,3,0]=-\frac{17}{9}, \quad U[1,0,4,0]=\frac{2}{3}, \\
U[3,0,4,0]=-\frac{17}{9}, \quad U[0,2,1,0]=-1, \ldots . .
\end{array}
$$

Using (3.3) and (4.2) and by recursive method, we get

$$
\begin{aligned}
& U[0,0,0,1]=-6, \quad U[0,0,1,1]=0, \quad U[0,1,0,1]=0, \quad U[1,0,0,1]=0, \\
& U[0,1,1,1]=12, \quad U[1,0,1,1]=12, \\
& U[1,1,0,1]=12, \quad U[1,1,1,1]=0, \quad U[0,0,2,1]=6, \quad U[0,2,0,1]=6, \\
& U[2,0,0,1]=6, \quad U[0,0,0,2]=0, \quad U[2,1,0,1]=0, \\
& U[0,1,2,1], \quad U[0,2,1,1], \quad U[2,0,1,1], \quad U[1,0,2,1], \ldots, \\
& V[0,0,0,1]=6, \quad V[0,0,1,1]=0, \quad V[0,1,0,1]=0, \\
& V[1,0,0,1]=0, \quad V[0,1,1,1]=-12, \quad V[1,0,1,1]=-12, \\
& V[1,1,0,1]=-12, \quad V[1,1,1,1]=0, \quad V[0,0,2,1]=-6, \quad V[0,2,0,1]=-6 \text {, } \\
& V[2,0,0,1]=-6, \quad V[0,0,0,2]=0, \\
& V[2,1,0,1]=0, \quad V[0,1,2,1]=0, \quad V[0,2,1,1]=0, \quad V[1,0,2,1]=0, \\
& V[1,2,0,1]=0, \quad V[1,2,1,1]=48, \quad V[2,1,1,1]=-16, \\
& V[2,2,0,1]=24, \quad V[2,0,2,1]=24, \quad V[1,1,2,1]=-16, \\
& V[0,2,2,1]=24, \quad V[2,2,1,1]=-64, \quad V[1,2,2,1]=-64, \ldots
\end{aligned}
$$


Substituting all $U(k, h, m, n)$ and $V(k, h, m, n)$ into (1.2) yields

$$
\begin{aligned}
& u(x, y, z, t)=1-6 t+x+20 t^{2} x+6 t x^{2}-56 t^{2} x^{2}-\frac{x^{3}}{3}-4 t x^{4}+y+20 t^{2} y+12 t x y-x^{2} y+\frac{2 x^{4} y}{3} \\
& +6 t y^{2}-56 t^{2} y^{2}-x y^{2}-24 t x^{2} y^{2}+\frac{4 x^{3} y^{2}}{3}-\frac{y^{3}}{3}+\frac{4 x^{2} y^{3}}{3}-\frac{17 x^{4} y^{3}}{9}-4 t y^{4}+\frac{2 x y^{4}}{3} \\
& -\frac{17 x^{3} y^{4}}{9}+z+20 t^{2} z+12 t x z-x^{2} z+\frac{2 x^{4} z}{3}+12 t y z-48 t^{2} y z-2 x y z+16 t x^{2} y z \\
& +\frac{8}{3} x^{3} y z-y^{2} z-48 t x y^{2} z+4 x^{2} y^{2} z-64 t x^{2} y^{2} z-\frac{17}{3} x^{4} y^{2} z-16 t y^{3} z+\frac{8}{3} x y^{3} z \\
& +\frac{2 y^{4} z}{3}-\frac{17}{3} x^{2} y^{4} z+6 t z^{2}-56 t^{2} z^{2}-x z^{2}-24 t x^{2} z^{2}+\frac{4 x^{3} z^{2}}{3}-y z^{2}+16 t x y z^{2} \\
& +4 x^{2} y z^{2}-128 t x^{2} y z^{2}-\frac{17}{3} x^{4} y z^{2}-24 t y^{2} z^{2}+4 x y^{2} z^{2}-64 t x y^{2} z^{2}+204 t x^{2} y^{2} z^{2} \\
& -\frac{34}{3} x^{3} y^{2} z^{2}+\frac{4 y^{3} z^{2}}{3}-\frac{34}{3} x^{2} y^{3} z^{2}-\frac{17}{3} x y^{4} z^{2}-\frac{z^{3}}{3}+\frac{4 x^{2} z^{3}}{3}-\frac{17 x^{4} z^{3}}{9}-16 t y z^{3}+\frac{8}{3} x y z^{3} \\
& +\frac{4 y^{2} z^{3}}{3}-\frac{34}{3} x^{2} y^{2} z^{3}-\frac{17 y^{4} z^{3}}{9}-4 t z^{4}+\frac{2 x z^{4}}{3}-\frac{17 x^{3} z^{4}}{9}+\frac{2 y z^{4}}{3}-\frac{17}{3} x^{2} y z^{4} \\
& -\frac{17}{3} x y^{2} z^{4}-\frac{17 y^{3} z^{4}}{9}+\cdots
\end{aligned}
$$

$$
\begin{aligned}
v(x, y, z, t)= & +6 t-x-20 t^{2} x-6 t x^{2}-56 t^{2} x^{2}+\frac{x^{3}}{3}+4 t x^{4}-y-20 t^{2} y-12 t x y+x^{2} y-\frac{2 x^{4} y}{3}-6 t y^{2} \\
& -56 t^{2} y^{2}+x y^{2}+24 t x^{2} y^{2}-\frac{4 x^{3} y^{2}}{3}+\frac{y^{3}}{3}-\frac{4 x^{2} y^{3}}{3}+\frac{17 x^{4} y^{3}}{9}+4 t y^{4}-\frac{2 x y^{4}}{3}+\frac{17 x^{3} y^{4}}{9} \\
& -z-20 t^{2} z-12 t x z+x^{2} z-\frac{2 x^{4} z}{3}-12 t y z-48 t^{2} y z+2 x y z-16 t x^{2} y z-\frac{8}{3} x^{3} y z \\
& +y^{2} z+48 t x y^{2} z-4 x^{2} y^{2} z-64 t x^{2} y^{2} z+\frac{17}{3} x^{4} y^{2} z+16 t y^{3} z-\frac{8}{3} x y^{3} z-\frac{2 y^{4} z}{3} \\
& +\frac{17}{3} x^{2} y^{4} z-6 t z^{2}-56 t^{2} z^{2}+x z^{2}+24 t x^{2} z^{2}-\frac{4 x^{3} z^{2}}{3}+y z^{2}-16 t x y z^{2}-4 x^{2} y z^{2} \\
& -128 t x^{2} y z^{2}+\frac{17}{3} x^{4} y z^{2}+24 t y^{2} z^{2}-4 x y^{2} z^{2}-64 t x y^{2} z^{2}-204 t x^{2} y^{2} z^{2}+\frac{34}{3} x^{3} y^{2} z^{2} \\
& -\frac{4 y^{3} z^{2}}{3}+\frac{34}{3} x^{2} y^{3} z^{2}+\frac{17}{3} x y^{4} z^{2}+\frac{z^{3}}{3}-\frac{4 x^{2} z^{3}}{3}+\frac{17 x^{4} z^{3}}{9}+16 t y z^{3}-\frac{8}{3} x y z^{3}-\frac{4 y^{2} z^{3}}{3} \\
& +\frac{34}{3} x^{2} y^{2} z^{3}+\frac{17 y^{4} z^{3}}{9}+4 t z^{4}-\frac{2 x z^{4}}{3}+\frac{17 x^{3} z^{4}}{9}-\frac{2 y z^{4}}{3}+\frac{17}{3} x^{2} y z^{4} \\
& +\frac{17}{3} x y^{2} z^{4}+\frac{17 y^{3} z^{4}}{9}+\cdots,
\end{aligned}
$$


Table 4: Exact and approximate solutions and absolute errors of $u(x, y, z, t),(y=z=0.01)$.

\begin{tabular}{lcccc}
\hline$x$ & $t$ & Exact solutions & Approximate solutions with DTM & Absolute errors \\
\hline 0.01 & 0.01 & 0.970008996761179 & 0.970102829745246 & 0.00009383 \\
0.02 & 0.02 & 0.920170230888868 & 0.920475254085735 & 0.00030502 \\
0.03 & 0.03 & 0.870727416393941 & 0.871248374408655 & 0.00052095 \\
0.04 & 0.04 & 0.821919131882669 & 0.822542425564273 & 0.00062329 \\
0.05 & 0.05 & 0.773971647721329 & 0.774464061599229 & 0.00049241 \\
0.06 & 0.06 & 0.727094919436867 & 0.727106307756535 & 0.00001138 \\
0.07 & 0.07 & 0.681479223097229 & 0.680548512475579 & 0.00093071 \\
0.08 & 0.08 & 0.637292532421948 & 0.634856299392122 & 0.00243623 \\
0.09 & 0.09 & 0.594678691310537 & 0.590081519338295 & 0.00459717 \\
0.10 & 0.10 & 0.553756389751220 & 0.546262202342606 & 0.00749418 \\
\hline
\end{tabular}

Table 5: Exact and approximate solutions and absolute errors of $v(x, y, z, t),(y=z=0.01)$.

\begin{tabular}{lcccc}
\hline$x$ & $t$ & Exact solutions & Approximate solutions with DTM & Absolute errors \\
\hline 0.01 & 0.01 & 1.0299910032388202 & 1.0298928497427533 & 0.000098153 \\
0.02 & 0.02 & 1.0798297691111314 & 1.0794940223302638 & 0.000335747 \\
0.03 & 0.03 & 1.1292725836060584 & 1.1286320940713441 & 0.00064049 \\
0.04 & 0.04 & 1.1780808681173303 & 1.1771196278117255 & 0.00096124 \\
0.05 & 0.05 & 1.226028352278671 & 1.2247558872007704 & 0.00127247 \\
0.06 & 0.06 & 1.2729050805631328 & 1.2713268846914636 & 0.00157820 \\
0.07 & 0.07 & 1.318520776902771 & 1.316605429540419 & 0.00191535 \\
0.08 & 0.08 & 1.362707467578051 & 1.3603511758078772 & 0.00235629 \\
0.09 & 0.09 & 1.4053213086894631 & 1.4023106703577044 & 0.00301064 \\
0.10 & 0.10 & 1.4462436102487797 & 1.4422174008573931 & 0.00402621 \\
\hline
\end{tabular}

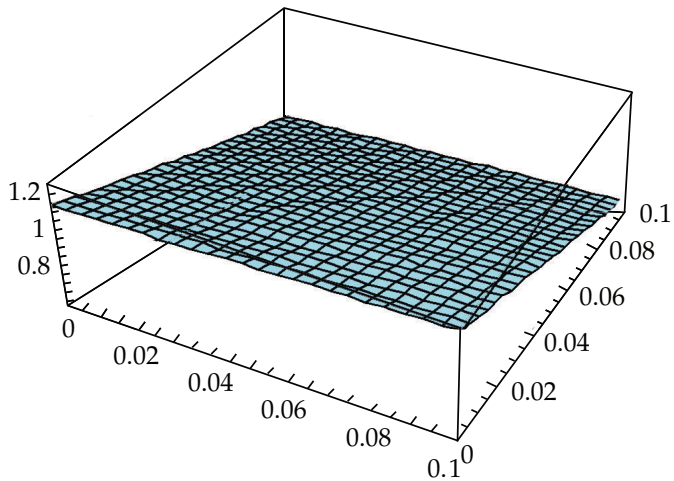

Figure 1: The analytic solution of $u(x, y, z, t)$.

and the analytical solution of the problem by Tanh method is

$$
\begin{aligned}
& u(x, y, z, t)=1+\operatorname{Tanh}(x+y+z-6 t) \\
& v(x, y, z, t)=1-\operatorname{Tanh}(x+y+z-6 t)
\end{aligned}
$$




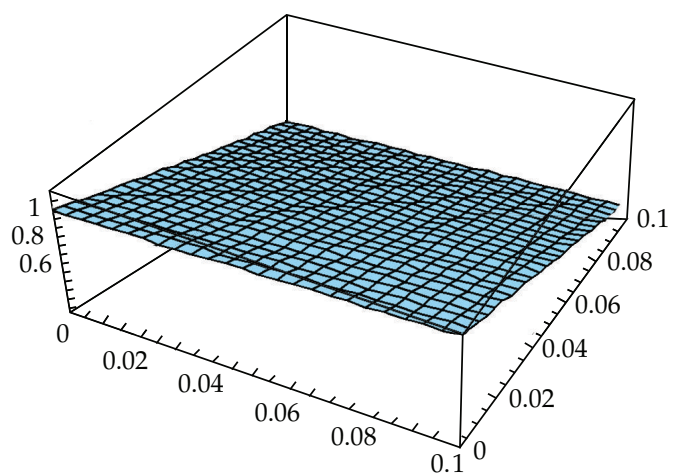

Figure 2: The approximate solution with DTM of $u(x, y, z, t)$.

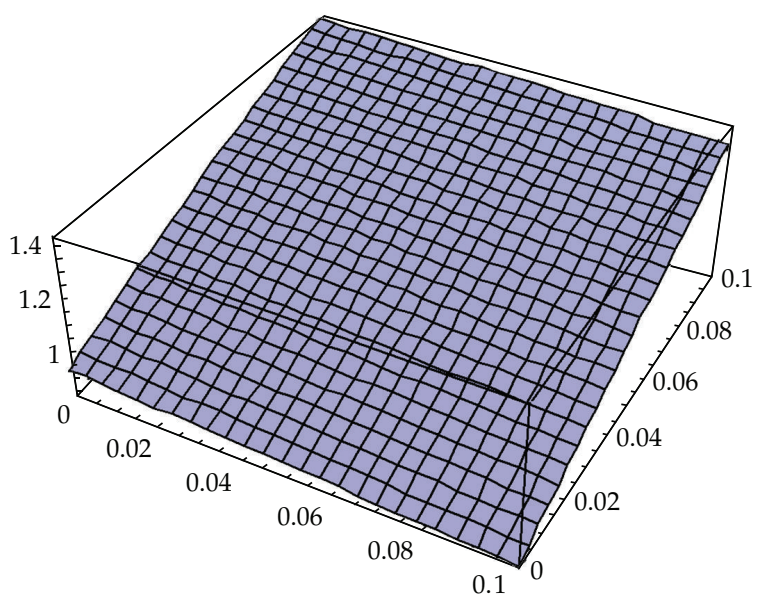

Figure 3: The analytic solution of $v(x, y, z, t)$.

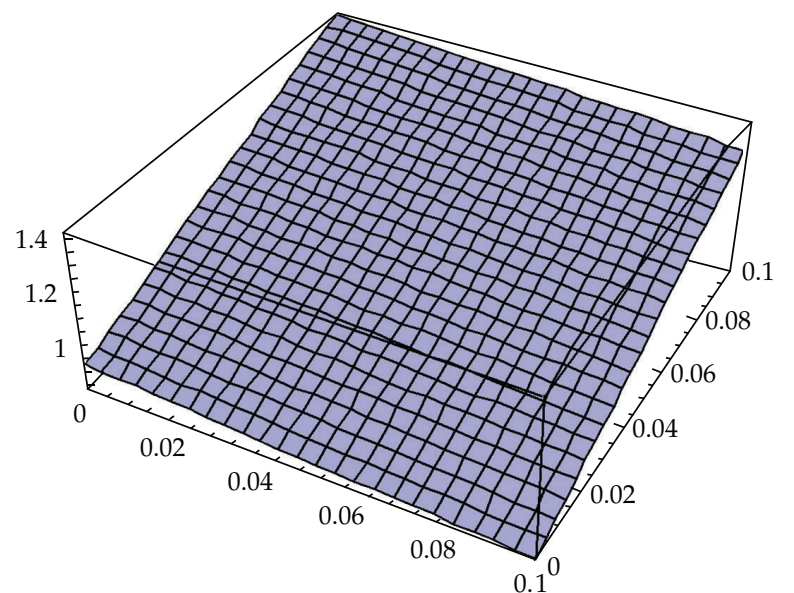

Figure 4: The approximate solution with DTM of $v(x, y, z, t)$. 


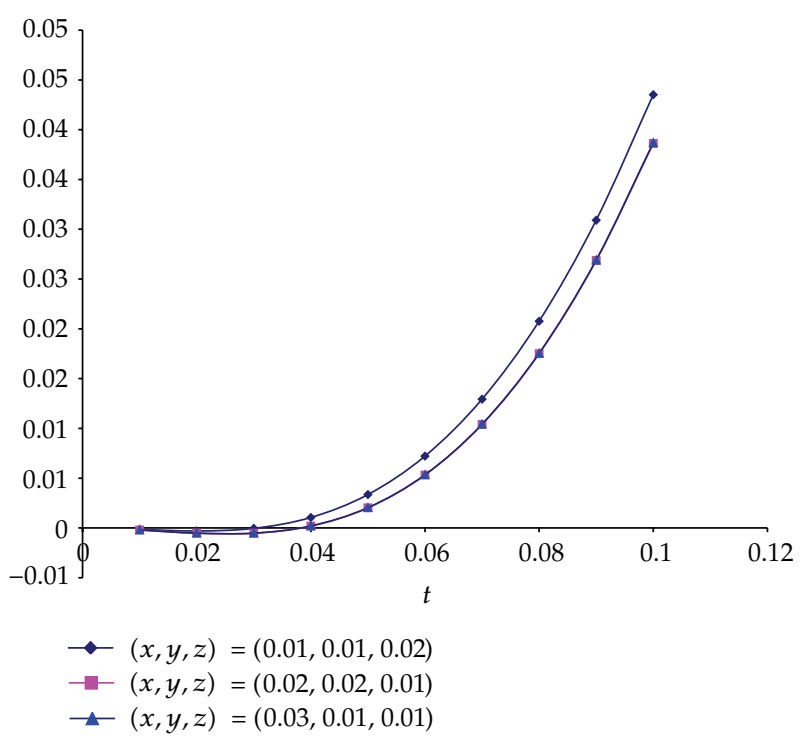

Figure 5: The error of $u_{\text {exact }}-u_{\mathrm{DTM}}$.

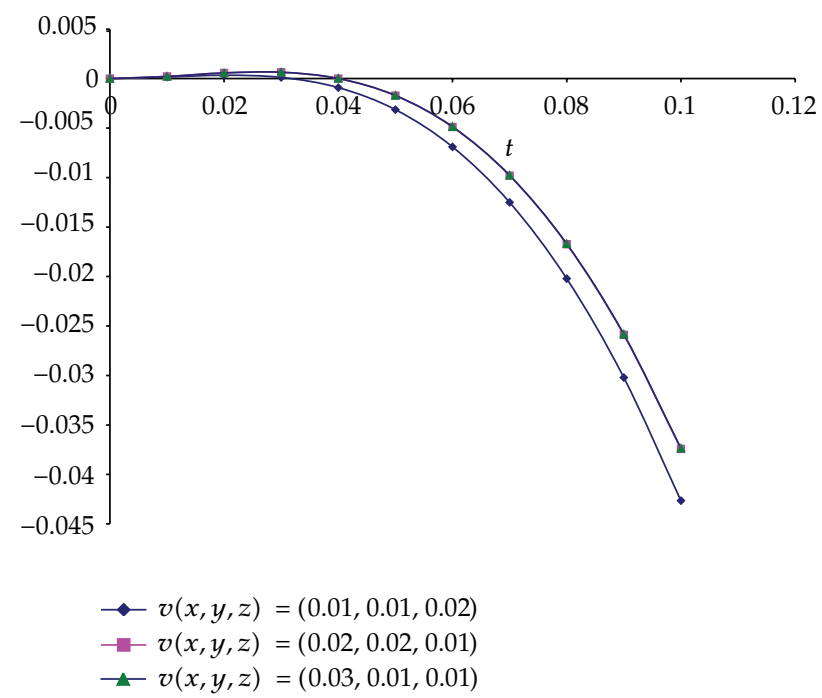

Figure 6: The error of $v_{\text {exact }}-v_{\text {DTM }}$.

When (4.4), (4.5), and (4.6) are compared, it can be seen that these two results are quite compatible for small values of $t$. For example, for $x=0.1, y=0.01, z=0.01$, and $t=0.001$, analytic solution of $u(x, y, z, t)$ is 1.1135087 and transform solution is 1.1135137 , and analytic solution of $v(x, y, z, t)$ is 0.886491 and transform solution is 0.886485 . For $x=0.1, y=0.01$, $z=0.01$, and $t=0.0001$, analytic solution of $u(x, y, z, t)$ is 1.1188358 and transform solution is 1.1188345, and analytic solution of $v(x, y, z, t)$ is 0.8811642 and transform solution is 0.8811654 (Tables 4 and 5, Figures 1 to 6). 


\section{Conclusion}

Four-dimensional differential transform has been applied to nonlinear system of PDEs. Analytic solution and transform solution are compared; the results are quite compatible for small values of $t$. The present method reduces the computational difficulties of the other methods, and all the calculations can be made by simple manipulations. On the other hand, the results are quite reliable. Therefore, this method can be applied to many complicated linear and nonlinear PDEs and system of PDEs and does not require linearization, discretization, or perturbation.

\section{Acknowledgments}

This paper was funded by the Deanship of Scientific Research (DSR), King Abdulaziz University, Jeddah, under Grant no. (68-130-D1432). The authors, therefore, acknowledge with thanks DSR technical and financial support.

\section{References}

[1] A. M. Wazwaz, "The decomposition method applied to systems of partial differential equations and to the reaction-diffusion Brusselator model," Applied Mathematics and Computation, vol. 110, no. 2-3, pp. 251-264, 2000.

[2] J. K. Zhou, Differential Transformation and Its Application for Electrical Circuits, Huazhong University Press, Wuhan, China, 1986.

[3] C. Kuang Chen and S. Huei Ho, "Solving partial differential equations by two-dimensional differential transform method," Applied Mathematics and Computation, vol. 106, no. 2-3, pp. 171-179, 1999.

[4] M. J. Jang, C. L. Chen, and Y. C. Liu, "Two-dimensional differential transform for partial differential equations," Applied Mathematics and Computation, vol. 121, no. 2-3, pp. 261-270, 2001.

[5] F. Ayaz, "On the two-dimensional differential transform method," Applied Mathematics and Computation, vol. 143, pp. 361-374, 2003.

[6] F. Ayaz, "Applications of differential transform method to differential-algebraic equations," Applied Mathematics and Computation, vol. 152, no. 3, pp. 649-657, 2004.

[7] F. Ayaz, "Solutions of the system of differential equations by differential transform method," Applied Mathematics and Computation, vol. 147, no. 2, pp. 547-567, 2004. 


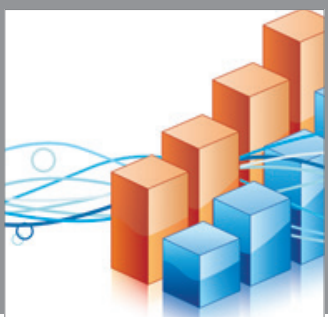

Advances in

Operations Research

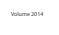

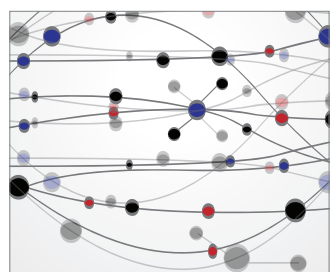

\section{The Scientific} World Journal
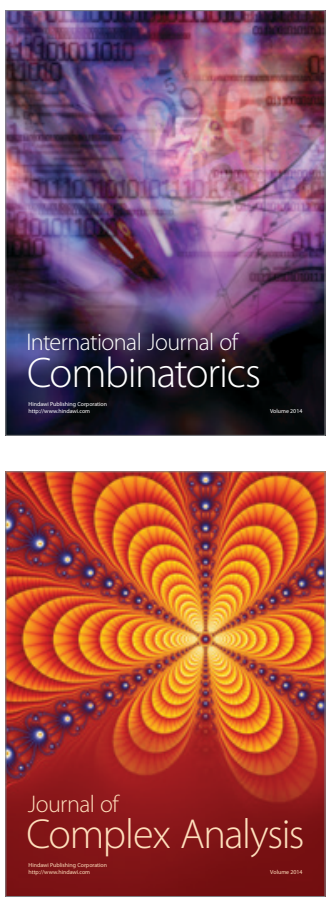

International Journal of

Mathematics and

Mathematical

Sciences
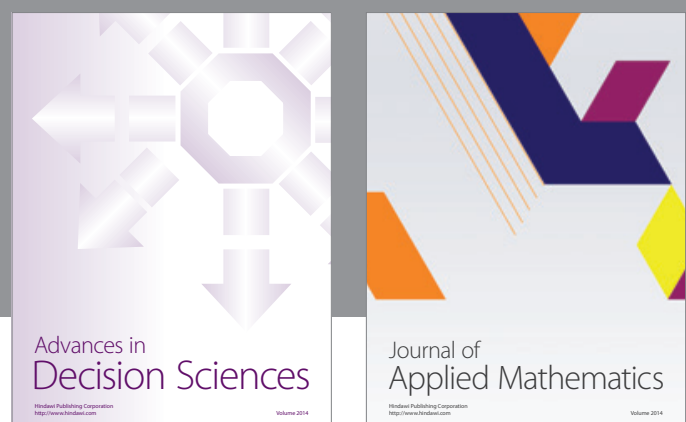

Journal of

Applied Mathematics
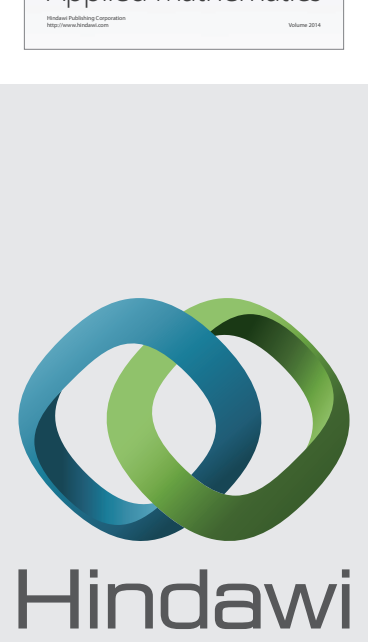

Submit your manuscripts at http://www.hindawi.com
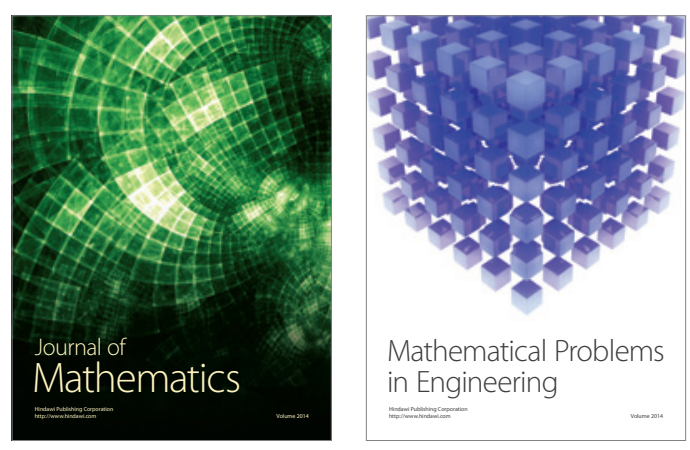

Mathematical Problems in Engineering
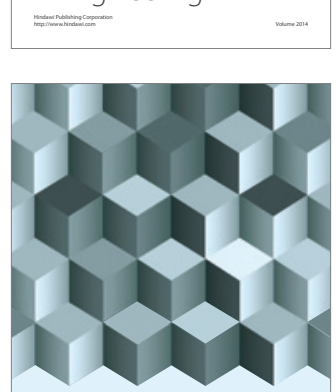

Journal of

Function Spaces
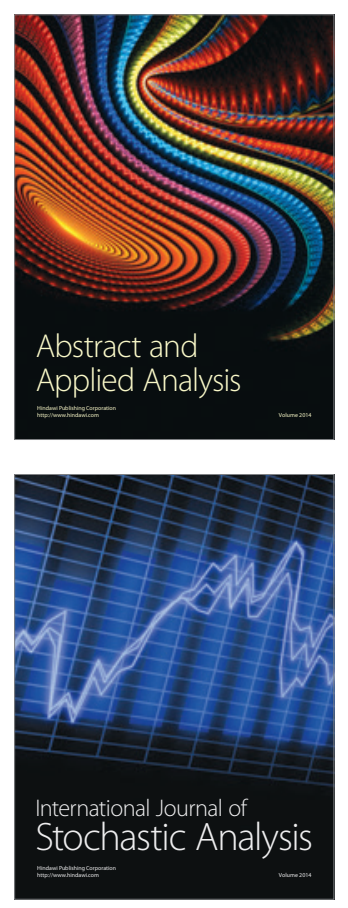

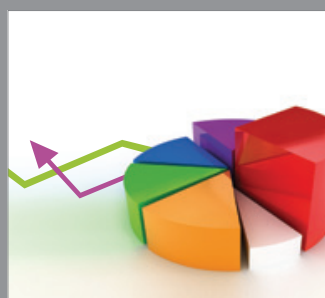

ournal of

Probability and Statistics

Promensencen
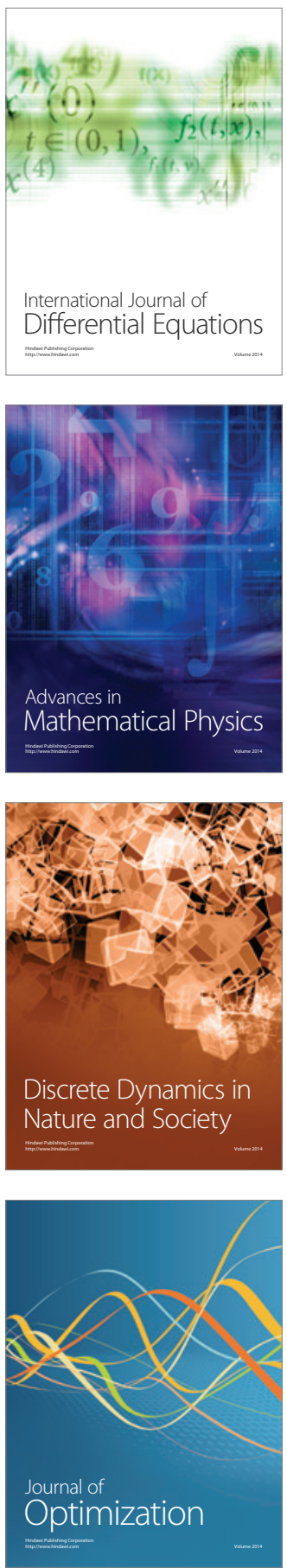